\title{
State-dependent learning with centrally and noncentrally active drugs
}

\author{
DANNIEL J. DOWNEY \\ University of Arizona, Tuscon, Arizona 85721
}

\begin{abstract}
State-dependent learning has been studied in cases where differential responses are required under varying drug conditions and where some unitary response must be performed under varying conditions of drug and nondrug. In the former case, response dissociation is considered to be the result of the cue properties of drugs, while in the latter case it is believed to be a consequence of CNS modification of the neural circuits representing coded responses. The present study attempted to determine if the stimulus properties of a drug without direct CNS activities could produce dissociation in a nondifferential responses (conditioned avoidance) task. Results indicated that only with the centrally active agents chlorpromazine $(2 \mathrm{mg} / \mathrm{kg})$ and pentobarbital $(20 \mathrm{mg} / \mathrm{kg})$ could any appreciable dissociation be obtained. No dissociation was obtained with the ANS stimulant, 1-epinepherine, at dosages of either .125 mg/kg or $.75 \mathrm{mg} / \mathrm{kg}$. It, thus, appears that the production of state-dependent learning involving a unitary response depends on direct action of a drug on the CNS and not simply on the occurrence of some spectrum of peripheral nervous system reactions.
\end{abstract}

Experiments attempting to demonstrate the occurrence of state-dependent learning effects with drugs have involved the use of two general procedures: (a) studies in which subjects are required to make spatial discriminations with the correct responses being cued by internal, state- (drug-) produced stimuli and (b) studies in which CNS states are modified by drug administration in testing phases in tasks not requiring differential response performance, such as with the conditioned avoidance response (CAR). The former procedures have led to the formulation of a drug-cue hypothesis which assumes that subjects utilize primarily the peripheral nervous system effects of drugs as discriminative stimuli for response evocation. The latter procedures, on the other hand, have fostered the development of a chemical milieu hypothesis (Overton, 1966) which says that state dependency represents the ability of the CNS to encode learned responses under the specific chemical conditions present during acquisition and to reproduce those responses only under sufficiently similar conditions.

Some investigators (e.g., Belleville, 1964; Otis, 1964) have attempted to apply the drug-cue hypothesis to cases in which state-dependent learning occurs but in which differential responding is not required. However, in these situations, the stimuli which adequately control the response are external stimuli which do not change from training to testing sessions. Internally produced cues, although present, are redundant and need not contribute significantly to the state-dependent effect. In such cases, the application of a drug-cue interpretation is unwarranted and perhaps inappropriate. The present study was designed in an attempt to determine the role of central vs. noncentral nervous system activity of a drug in its ability to produce state-dependent

This paper is sponsored by J. E. Lyons who takes full editorial responsibility for it. dissociation. If peripheral cues and their utilization are the important factors in state-dependency, then evidence of dissociation should be obtained with the noncentrally active agent, 1-epinephrine, even when a nondifferential response task is used. However, if, as the chemical milieu hypothesis suggests, CNS modification is necessary, then dissociation should be obtained only with the centrally active agents pentobarbital and chlorpromazine.

\section{METHOD}

\section{Subjects}

Eighty male albino rats (Holtzman strain) weighing from $350-400 \mathrm{~g}$ throughout the entire experiment were used as subjects. The animals were housed individually in wire mesh cages and food and water were available ad lib throughout the duration of the experiment.

\section{Apparatus}

The apparatus consisted of a standard two-compartment shuttlebox measuring $24 \times 10.5 \times 10$ in. and constructed of wood, reinforced fiberboard, and Plexiglas panels. The shuttlebox floor was constructed of 1/16-in diam. copper rods placed approximately $1 / 4$ in. apart and the apparatus itself was divided into two equal sized compartments by an electrified 3-in. high hurdle.

Shock was produced by a scrambler-shock generator containing variable voltage controls allowing the experimenter to adjust shock levels during training for individual subjects. The location of shock was programmed automatically by microswitches attached to the grid floors of the two compartments. Intertrial intervals, shock duration, and CS duration were regulated by two Hunter decade interval timers, and latency data were recorded atuomatically by a multiactivity printout counter (Layfayette Instrument Company). The only illumination provided during the experiment was produced by a 25 -W cylindrical bulb located approximately 15 in. above the top of the apparatus.

\section{Procedures}

Subjects were randomly assigned to one of 16 groups, 5 subjects per group. The experimental design involved the use of a 
simple switchback, transfer-of-training paradigm in which all four possible combinations of drug (D) and nondrug (ND) were represented. This yielded four groups (ND-ND, D-ND, ND-D, and D-D) for each of four drugs, for a total of 16 groups. Drug states were produced by injection of chlorpromazine hydrochloride (CPZ; Smith, Kline and French), $2 \mathrm{mg} / \mathrm{kg}$; sodium pentobarbital (SPe; Abott), $20 \mathrm{mg} / \mathrm{kg}$; or 1-epinephrine (Epi; J. $\mathrm{H}$. Walker, $.125 \mathrm{mg} / \mathrm{kg}$ or $.75 \mathrm{mg} / \mathrm{kg}$. SPe and CPZ and their dosages were chosen on the basis of past research which has shown them to be state-dependency-producing drugs (Overton, 1966; Miller, 1961) and as referents against which cathecholamine effects could be evaluated. Epi was chose at dosages known to be behaviorally active in the CAR situation (Latané \& Schacter, 1961) and for its ability to produce a wide range of autonomic system effects without crossing the blood brain barrier to any appreciable extent (Weil-Malherbe, Axelrod, $\&$ Tomchick, 1959). Nondrug states were provided for by injections of normal saline $(.9 \% \mathrm{NaCl})$ in amounts equal to those of drug volumes for each individual subject.

$\mathrm{CPZ}$, saline, and SPe were injected in traperiotoneally in equal volumes (range $=.25-.30 \mathrm{cc}$ ) $45 \mathrm{~min}$ prior to the day's session. Epi was suspended in peanut oil and injected subcutaneously into the nape of the neck $1.5 \mathrm{~h}$ prior to the day's session. In the case of SPe, it was necessary for the animal to recover sufficiently from the ataxic effects of the drug before testing could be conducted; this usually required $30-45$ min depending on the individual subject.

Phase I (training) for all subjects consisted of six daily sessions, 20 trials per day, one session per day, on consecutive days. A trial was defined as either a single avoidance or escape response and consisted of CS (damped house buzzer) presentation for $7 \mathrm{sec}$, followed by UCS (shock) with the CS terminating simultaneously with UCS onset in all cases. If a response was made prior to UCS onset, the CS was terminated and the UCS eliminated for that trial. The intertrial interval was $15 \mathrm{sec}$ in length and responses during this interval were never specifically punished.

In Phase II (testing), subjects were given a chance to perform the CAR under the same procedures described above for Phase I, except that appropriate state-change or no state-change conditions were now in operation. The test phase consisted of 20 trials per session, for two sessions on 2 consecutive days, one session per day. Thus, for subjects performing under conditions identical to those of training (no state-change) or for subjects not demonstrating the dissociation effect, Phase II should serve as additional training time. On the other hand, for subjects in state-change groups demonstrating the dissociation effect, Phase II performance should decrease and the testing phase should act as a period of reacquisition.

\section{RESULTS}

Drug effects on the acquisition of the CAR task were evaluated by use of a 6 by 5 (days of acquisition vs. groups) mixed design analysis of variance for both latency and percentage CAR data (see Figure 1). Since saline subjects were run in four groups of 10 subjects each and since there were no differences between the four runs, saline acquisition were represented by that of the first 10 saline subjects. Results indicated that there was a significant difference for both the groups ( $F s=$ $13.39, \mathrm{p}<.001 ; 6.62, \mathrm{p}<.005$ for CAR and latency data, respectively) and days factors ( $F s=110.08$, $\mathrm{p}<.001 ; 103.64, \mathrm{p}<.001)$. Post hoc tests using the

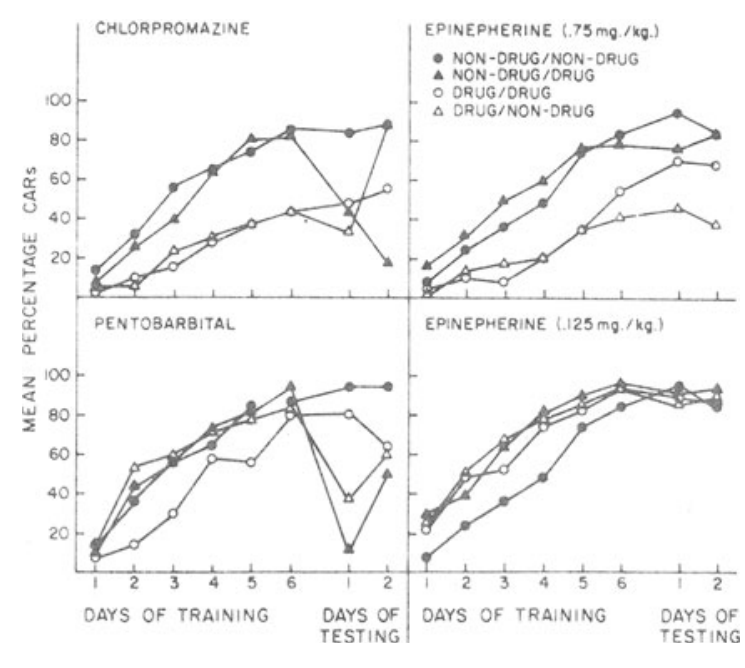

Figure 1. Percentage conditioned avoidance responses made during acquisition training and state-changing testing.

Newman-Kuels procedures revealed that on both measures these results were due to acquisition decrements in the CPZ $(p<.05)$ and Epi $.75 \mathrm{mg} / \mathrm{kg}$ $(p<.05)$ groups and that these decrements were reliable on Days 3-6 ( $p<.05$ for each).

State-dependent effects were evaluated by use of separate 2 by 2 analysis of variance (drug or no-drug in training vs, state-change or no state-change in testing) for each drug considered on each of the 2 days of testing. The initial analyses were then followed by post hoc tests using the Newman-Keuls procedure, which compared the ND-D and D-ND state-change groups with their controls, ND-ND and D-D, respectively. This procedure was used so that the major source of dissociation could be more adequately assessed. Thus, if the comparison between the D-ND and D-D groups was not significant and that between the ND-ND and ND-D groups was, then the state change from ND to $\mathrm{D}$ would be contributing maximally to the dissociation effect and this would be interpretable as a simple drug effect.

Figure 1 depicts the performance of all 16 groups in both training and testing phases of the experiment. The lower left hand panel shows the large deficits in performance with pentobarbital in state-change groups on Day 1 , of testing which amounts to average decrements of $82 \%$ in the ND-D group and $48 \%$ in the D-ND group. This lower mean decrement in the latter group is the result of a single subject for whom no dissociation was evidenced, the remaining four subjects of that group having decrements of 50\%-85\%. Analysis of variance indicated significant state-change effects $(\mathrm{F}=$ $47.38, p<.001$ for CAR data and $F-33.23, p<.001$ for latency data) and post hoc comparisons revealed that both state-changes have significant decrements when 
compared to their respective controls $(p<.001$ for each). On Day 2 of testing, increments in both state-change groups occur reducing average performance decrements compared to final performance in training to $24 \%$ in the D-ND group and $45 \%$ in the ND-D group. Decrements in responding were still substantial in three subjects in the D-ND group while only two subjects in the ND-D group continued to respond poorly. Analysis of variance indicated a state-change effect at a reduced level of statistical significance $(F=3.13, p<.10$ and 4.03, $\mathrm{p}<.01$ for CAR and latency data, respectively) and post hoc comparisons again revealed that both conditions contribute to the effect.

As can be seen in the upper left, CPZ panel of Figure 1, no state-change groups continue to perform as they did late in training while state-change groups show decrements on Day 1 of testing. These changes in performance amount to an average of $37 \%$ and $11 \%$ in the ND-D and D-ND groups, respectively. In the important D-ND group, although the dissociation effect for $\mathrm{CPZ}$ is small compared to that obtained with pentobarbital, 4 of 5 animals demonstrated the decrements in responding and these ranged from 5\% to $30 \%$. Statistical analyses indicated significant state-change effects using both percentage CAR and latency data. In the case of CPZ however, post hoc tests of Day 1 data revealed that comparisons of ND-ND and ND-D groups were significant $(\mathrm{p}<.05)$ while those for D-D and D-ND groups were not. On Day 2, group ND-D showed even larger decrements while Group D-ND's performance improved dramatically, equaling that of nondrugged, no state-change subjects. In effect, whatever dissociation effects were present in the latter group on Day 1 were now absent.

It is evident from the data in the right-hand panels of Figure 1 that no state-dependent dissociation was obtained at either of the two Epi dosage levels. With the $.125 \mathrm{mg} / \mathrm{kg}$ dose, acquisition is slightly, but not significantly, better than that of controls and average decrements amount to as little as $0-6 \%$ in state-change groups. At the higher dosage, despite reliable decrements in acquisition, state-change groups' performance is not disrupted on testing days, average decrements ranging from $0 \%-5 \%$. Analyses of variance indicated no significant effect of state-change at either dosage level, on either day of testing, for either percentage CAR or latency response measures.

\section{DISCUSSION}

In the present experiment, state-dependent dissociation was obtained with the centrally active agents $\mathrm{CPZ}$ and $\mathrm{SPe}$, but not with the noncentrally active Epi at either high or low dosages. Dissociation was greatest in the case of SPe and the degree of dissociation obtained in the present experiment compares favorably with that evidenced in previous research with barbiturates and both state-dependent response control (Overton, 1964, 1966) and state-dependent dissociation (Barry, Etheredge, \& Miller, 1965; Bindra, Nyman, \& Wise, 1965; Bindra \& Reichert, 1966, 1967). Although CPZ has reported to produce state-dependent dissociation ( $\mathrm{Lal}, 1969$; Otis, 1964), the effect is typically small in magnitude compared to that produced by other centrally active depressants. In the present case, some degree of dissociation was obtained with $\mathrm{CPZ}$ on Day 1 of testing; but this was primarily due to decrements in the ND-D group and what dissociation was present was eradicated by Day 2. Miller(1961) has likewise reported that state changes with $\mathrm{CPZ}$ yield small dissociative effects and that performance after the first test day for dissociation is almost identical to that of prechange days.

Neither the $.125 \mathrm{mg} / \mathrm{kg}$ nor $.75 \mathrm{mg} / \mathrm{kg}$ dosages of Epi were able to produce state-dependent dissociation. Despite the fact that Epi stimulated CAR acquisition at the low dosage and dramatically disrupted it at the high dosage, change of state had no apparent effect on behavior. Since Epi administration elicits a host of autonomic and somatic reactions but no dissociation, this would argue against the drug-cue interpretation of state-dependent learning. Apparently, modification of CNS states by direct drug action is a necessary condition for the production of response dissociation. Manipulations such as that used here may indeed alter the stimulus character of the environment, but without direct CNS action they are not effective in altering the encoding nervous system representation of the response.

The inability to produce state-dependent learning with epinephrine does not seem to be a result of the drug itself characteristically or of the dosages used in the present experiment. Cook, Davidson, Davis, and Kelleher (1960) have reported that subjects can be successfully reflex conditioned when the CS was internal, peripheral stimuli produced by 1 epinephrine $(10 \mathrm{ug} / \mathrm{kg}$, iv), 1-norepinephrine $(10 \mathrm{ug} / \mathrm{kg}$, iv), ace tylcholine $(10 \mathrm{ug} / \mathrm{kg}$, iv), or jejunal loop pressure $(10 \mathrm{~cm}-\mathrm{Hg})$. Thus, epinephrine and other peripherally active agents can produce state-dependent-like effects, but apparently only in cases where the physiological stimuli they produce are maximized for learning; that is, where they are the only cues available to the subject for determining what response is necessary.

Other lines of evidence from the response control literature also suggest that state-dependent learning depends on the modification of central nervous system states. Overton (1964) has reported that attempts to obtain state-dependent response control when the discriminative stimuli were injections of gallamine (a skeletal muscle relaxant) and a tetraethylammonium (an ANS blocking agent) or single sensory stimuli were either inefficient and/or unsuccessful. Moreover, manipulation of shock levels or the visual capacity of subjects did not affect the existent response control exerted by barbiturates, suggesting that changes in sensory reception were not involved in the production of dissociation (Overton, 1968). As Overton (1971, a, b) further points out, among the antimuscarinic and antinicotinic drugs used in studies of response control, only those which possess both peripheral and central nervous system activities are effective to any appreciable extent.

The foregoing discussion does not mean to imply that drug-produced, internal cues can never attain discriminative control over behavior-only that in tasks not maximizing the utilization of those cues for response evocation are they unimportant. At the least, for cases in which the occurrence of dissociation is applicable (i.e., those not involving differential responding), the neural basis of stored, learned responses must be modified-and, in the case of drugs, this required direct CNS activity.

\section{REFERENCES}

Barry, H., Etheredge, E., \& Miller, N. E. Counterconditioning and extinction of fear fail to trasfer from amobarbital to nondrug state. Psy chopharmacologia, 1965, 8, 150-156.

Belleville, R. E. Control of behavior by drug-produced internal stimuli. Psychopharmacologia, 1964, 5, 95-105.

Bindra, D., Nyman, K., \& Wise, J. Barbiturate-induced 
dissociation of acquisition and extinction: role of movement-initiating processes. Journal of Comparative and Physiological Psychology, 1965, 60, 223-228.

Bindra, D., \& Reichert, H. Dissociation of movement initiation without dissociation of response choice. Psychonomic Science, 1966, 4, 95-96.

Bindra, D., \& Reichert, H. The nature of dissociation: Effects of transitions between normal and barbiturate-induced states on reversal learning and habituation. Psy chopharmacologia, 1967, 10, 330-344.

Cook. L., Davidsion, A., Davis, D. J., \& Kelleher, R. T. Epinephrine, norepinephrine, and acetylcholine as conditioned stimuli for avoidance behavior. Science, 1960 , conditioned stin

Lal, H. Control of learned conditioned avoidance responses (CAR) by amphetamine and chlorpromazine. Psychopharmacologia, 1969, 14, 33-37.

Latane, B., \& Schacter, S. Adrenalin and avoidance learning. Journal of Comparative and Physiological Psychology, 1962, 55, 369-373.

Miller, N. E. Some recent studies of conflict behavior and drugs. American Psy chologist, 1961, 16, 12-24.
Otis, L. Dissociation and recovery of a response learnied under the influence of chlorpromazine or saline. Science, 1964, 143, 1347-1348.

Overton, D. State-dependent or dissociated learning produced with pentobarbital. Journal of Comparative and Physiological Psychology, 1964, 57, 3-12.

Overton, D. State-dependent learning produced by depressant and atropine-like drugs. Psy ch opharmacologia, 1966, 10, 6-31.

Overton, D. Visual cues and shock sensitivity in the control of T-maze choice by drug conditions. Journal of Comparative and Physiological Psychology, 1968, 66, 216-219.

Overton, D. Commentary. In Harvey, J. A. (Ed.), Behavioral analysis of drug action: Research and commentary. Illinois: Scott, Foreman, 1971a.

Overton, D. Discriminative control of behavior by drug states. In Thompson, T., \& Pickens, R., (Eds.), Stimulus properties of drugs. New York: Appleton-Centry-Crofts, $1971 \mathrm{~b}$.

Weil-Malherbe, H., Axelrod, J., \& Tomchick, R. Blood-brain-barrier 'for adrenaline. Science, 1959, 129, 1226-1227.

(Received for publication December 9, 1974.) 